\title{
Atypical crusted scabies in a patient with chronic liver disease caused by hepatitis B and D viruses*
}

\author{
Mônica Santos ${ }^{1,2}$ \\ Carolina Talhari ${ }^{1}$
}

\author{
Gustavo Ávila Maquiné ${ }^{1}$ \\ Antonio Pedro Mendes Schettini ${ }^{3}$
}

DOI: http:/ / dx.doi.org/10.1590/abd1806-4841.20187023

\begin{abstract}
Crusted scabies is a less common variant of scabies that is highly contagious, difficult to treat and involves infestation by Sarcoptes scabiei var. hominis. The classical clinical presentation includes crusted, scaly and generally non-pruritic lesions usually located on the head, neck, palmar, plantar and periungual region. It was first described in Norway in 1848 in patients with leprosy who presented with crusted lesions. In this study, we report the case of a patient with crusted scabies with florid clinical manifestations and chronic liver disease due to hepatitis B and delta virus infection.
\end{abstract}

Keywords: Hepatitis B; Hepatitis D; Scabies

\section{INTRODUCTION}

Crusted scabies (CS) is a less common variant of scabies with a massive infestation by Sarcoptes scabiei var. hominis, related to poor hygiene, institutionalization, immunosuppressive conditions or medications, psychiatric disturbances and sensory neuropathies as predisposing factors. ${ }^{1}$ The diagnosis of the disease is clinical; however, in cases of diagnostic uncertainty, microcopy of skin scrapings or histopathology can be performed. ${ }^{2}$ Treatment is preferentially with ivermectin $200 \mu \mathrm{g} / \mathrm{kg}$ on days 1, 2, 8, 9 and 15 . Permethrin $5 \%$ or Sulphur $5 \%$ associated with keratolytics can also be used. ${ }^{3}$

\section{CASE REPORT}

A 46-year-old male patient, born in Tapauá (AM) and living in Manaus (AM), attended the dermatology outpatient clinic with grey, scaly, well-defined plaques on the trunk, buttocks and lower limbs (Figure 1). His past history included motor neuropathy due to cerebral palsy. The patient also reported alcohol and tobacco consumption. Direct microscopy from a skin scraping revealed numerous parasites. Histopathology with H\&E revealed hyperkeratosis, parakeratosis, papillomatosis, pseudoepitheliomatous hyperplasia, focal intraepidermal eosinophilic infiltrate, besides mites in the stratum corneum (Figure 2). The patient also had peripheral
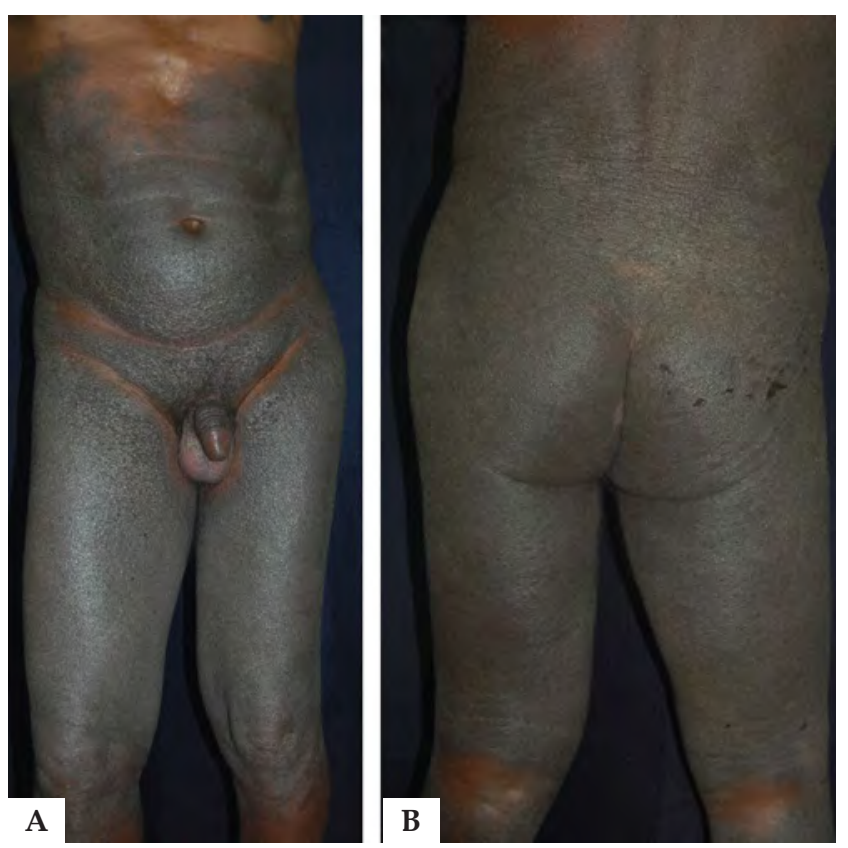

Figure 1: Presence of well-defined grayish scaly plaques on his trunk, genitals and thighs (A) and in the gluteal region (B)

Received on 20.02.2017.

Approved by the Advisory Board and accepted for publication on 28.04.2017.

* Study conducted at Fundação Alfredo da Matta (Fuam) - Manaus (AM), Brazil.

Financial support: None.

Conflict of interest: None.

Outpatient Clinic of Tropical Dermatology, Fundação Alfredo da Matta (Fuam) - Manaus (AM), Brazil.

Department of Dermatology, Universidade do Estado do Amazonas (UEA) - Manaus (AM), Brazil.

Service of Dermatopathology, Fundação Alfredo da Matta (Fuam) - Manaus (AM), Brazil.

MAILING ADDRESS:

Mônica Santos

E-mail: m.n.souza.santos@gmail.com 

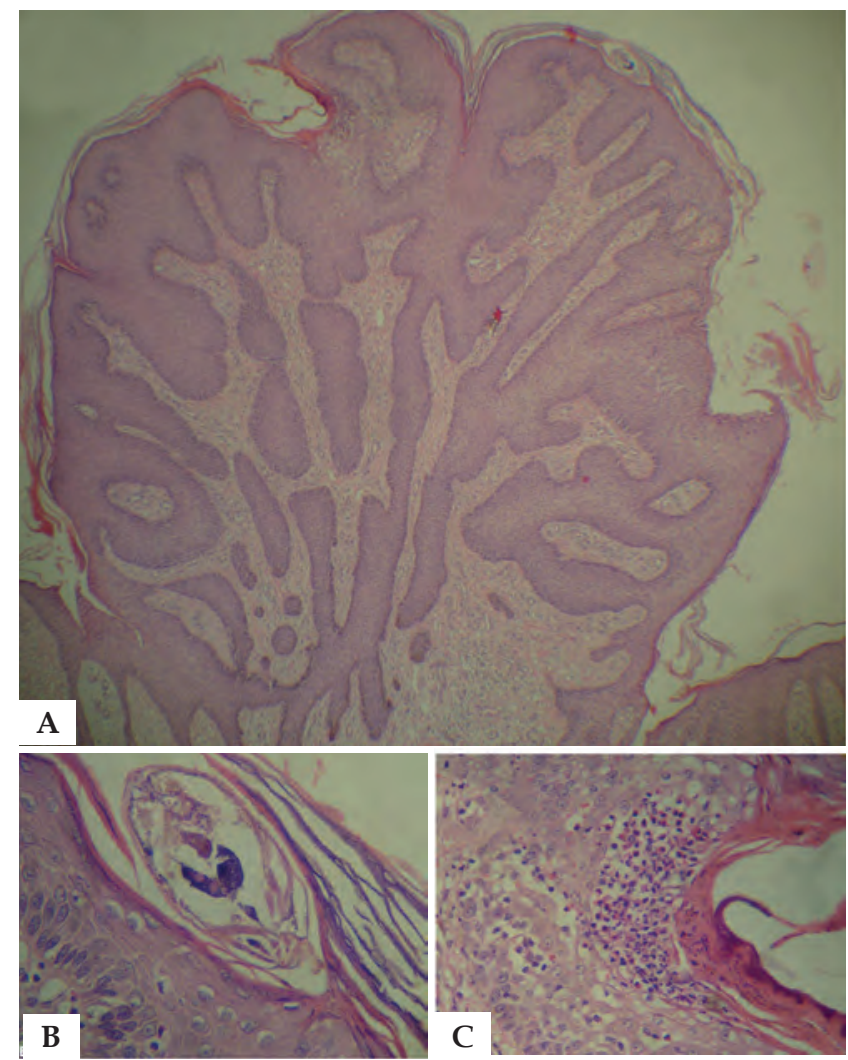

FIGURE 2: Hematoxylin \& eosin staining of histology sections revealed hyperkeratosis, parakeratosis, papillomatosis, pseudoepitheliomatous hyperplasia (A - Hematoxylin \& eosin, $\mathrm{X} 100)$, focal eosinophilic infiltrate in the epidermis and mites in the stratum corneum (B and C - Hematoxylin \& eosin, X400)

eosinophilia (23\%) and monoclonal IgE 20-times higher than normal (2500 U/ml). Serology was positive for hepatitis B and delta viruses, with positive $\mathrm{HBsAg}$, anti-HBc-T, anti-HBe and anti-HDV. HIV and HTLV serologies were negative. Abdominal ultrasound revealed signs of chronic liver disease. Treatment was performed with five doses of ivermectin $(200 \mu \mathrm{g} / \mathrm{kg} /$ day $)$ and Sulphur $5 \%$ associated to salicylic acid in Vaseline for 5 days. He progressed with clinical cure (Figure 3).
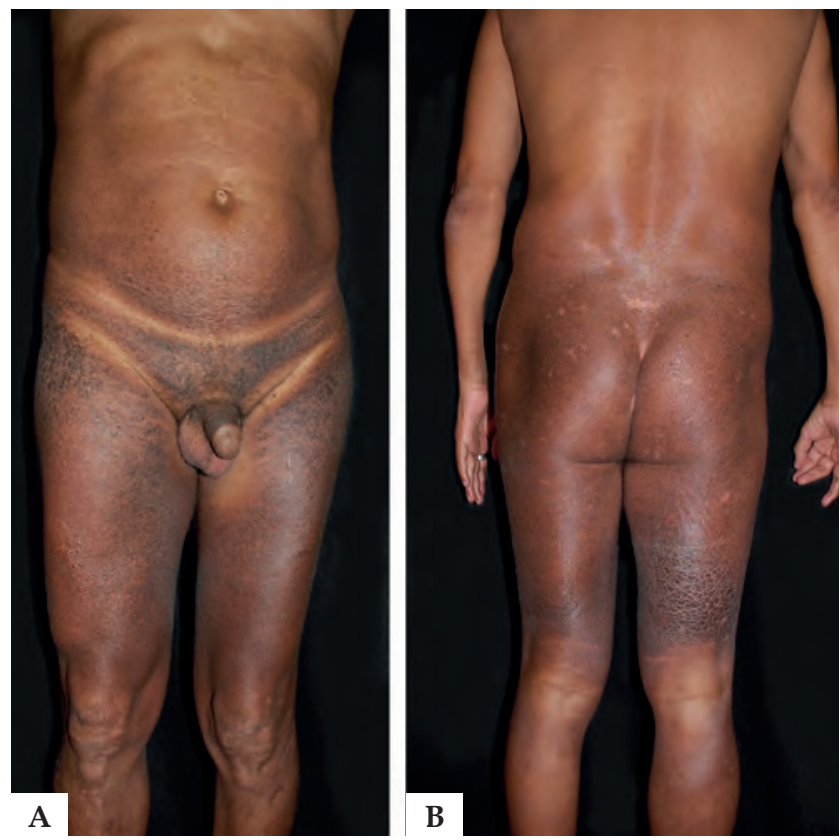

Figure 3: After treatment, the patient's clinical condition was resolved, remaining only residual hyperchromia

\section{DISCUSSION}

The mechanism for the development of CS is still not totally clear, but it is frequently associated to an imbalance between cellular and humoral immunities. There would be a predominance of the humoral response (T-helper 2) with raised levels of IgE, IgG and eosinophils and negligible cellular immune response in CS. The inability of controlling the parasite multiplication could explain the florid lesions. ${ }^{4}$ During laboratory screening, we observed that the patient had chronic liver disease due to hepatitis B and delta viruses, which, associated to chronic alcoholism could justify the reduced immunity and the appearance of an extensive and atypical form of CS. The patient was born in Tapauá, a municipality part of the hepatitis B and delta viruses endemic area in the Amazonas. ${ }^{5}$ The patient responded satisfactorily to the prescribed treatment, and is undergoing follow-up with the dermatology and hepatology clinics. $\square$

\section{REFERENCES}

1. Shmidt E, Levitt J. Dermatologic infestations. Int J Dermatol. 2012; 51:131-41. 2. Golant AK, Levitt JO. Scabies: a review of diagnosis and management based on mite biology. Pediatr Rev. 2012;33:e1-e12.

3. Shimose L, Munoz-Price LS. Diagnosis, prevention, and treatment of scabies. Curr Infect Dis Rep. 2013;15:426-31.
4. Walton SF. The immunology of susceptibility and resistance to scabies. Parasite Immunol. 2010;32:532-40.

5. Currie BJ, McCarthy JS. Permethrin and ivermectin for scabies. N Engl J Med. 2010;362:717-25.

How to cite this article: Santos M, Maquiné GA, Talhari C, Schettini APM. Atypical crusted scabies in a patient with chronic liver disease caused by hepatitis B and D viruses. An Bras Dermatol. 2018;93(2):277-8. 